\title{
Evolution of the tissue factor pathway inhibitor-like Kunitz domain-containing protein family in Rhipicephalus microplus
}

Elizabeth Louw ${ }^{\mathrm{a}}$, Nicolaas A. van der Merwe ${ }^{\mathrm{b}}$, Albert W. H. Neitz ${ }^{\mathrm{a}}$, Christine Maritz-Olivier ${ }^{\mathrm{b}, *}$

a Department of Biochemistry, Faculty of Natural and Agricultural Sciences, University of Pretoria, Lynnwood Road, Pretoria, South Africa, 0002

${ }^{\mathrm{b}}$ Department of Genetics, Faculty of Natural and Agricultural Sciences, University of Pretoria, Pretoria, South Africa, 0002

*Corresponding author.

Christine Maritz-Olivier, Room 7-30, Department of Genetics, Faculty of Natural and Agricultural Sciences, University of Pretoria, Lynnwood Road, Pretoria, South Africa 0002

Tel.: +27 124203945 / +27 12420 3258; fax: +27 123625327 / +27 123625302 .

E-mail address: christine.maritz@up.ac.za

\section{ABSTRACT}

One of the principle mechanisms utilised by ticks to obtain a blood meal is the subversion of the host's haemostatic response. This is achieved through the secretion of saliva containing antihaemostatic proteins into the feeding lesion. Lineage-specific expansion of predicted secretory protein families have been observed in all previously studied ticks and occurred in response to adaptation to a blood-feeding environment. Of these, the predominant families are common between both hard and soft ticks. One of these families, namely the Kunitz domain-containing protein family, includes proven tissue factor pathway inhibitor-like (TFPI-like) anti-haemostatics such as ixolaris and penthalaris that play a crucial role during tick feeding. Although Kunitz-type proteins have been found in Rhipicephalus microplus, the TFPI-like Kunitz protein family has not yet been studied. We report a comprehensive search for TFPI-like Kunitz domain-containing proteins in $R$. microplus expressed sequence tag libraries, resulting in the identification of 42 
homologues. The homologues were bioinformatically and phylogenetically studied, including the application of an intensive Bayesian Markov Chain Monte Carlo (MCMC) analysis of the individual Kunitz domain nucleotide sequences. We show that the $R$. microplus TFPI-like Kunitz protein family groups into two main clades that presumably underwent ancient duplication, which indicates that a whole genome duplication event occurred at least 150 million years ago. Evidence for recent and ancient gene and domain duplication events was also found. Furthermore, the divergence times of the various tick lineages estimated in this paper correspond with those presented in previous studies. The elucidation of this large protein family's evolution within $R$. microplus adds to current knowledge of this economically important tick.

Keywords:

Rhipicephalus microplus

Kunitz domain

Tissue factor pathway inhibitor (TFPI)

Phylogenetic

Bayesian Markov Chain Monte Carlo

Duplication

\section{Introduction}

Rhipicephalus microplus is considered to be the most important tick parasite of cattle worldwide, resulting in profit losses and financial expenditures due to both direct and indirect damage to the animals. Infestation by these ticks causes reductions in weight gain and milk production. This tick species is also responsible for indirect damage to cattle due to its vectoring capability for anaplasmosis and babesiosis pathogens. Losses due to R. microplus infestations have been estimated at US \$7 billion annually (Peconick et al., 2008) and these losses are expected to rise due to expansion of the tick's habitable range into non-endemic areas (White et al., 2003; 
Estrada-Peña et al., 2005; Lynen et al., 2008). Currently, only one tick vaccine has been developed and is marketed as Gavac in Central and South America, and as TickGARD in Australia (de la Fuente et al., 2007a). It is a recombinant vaccine based on a $R$. microplus gut antigen (Bm86) and while it has been successfully used in conjunction with other acaricides it exhibits limited and varying efficacy against different tick species and strains (reviewed by Willadsen, 2004). Therefore, the identification of novel tick vaccine candidates is of great importance to the global cattle industry.

Recently, tick research has benefitted greatly from genetic technologies such as expressed sequence tag (EST) studies and whole-genome sequencing. Before the advent of such technologies, gene discovery had been achieved through time-consuming and laborious experiments, often resulting in the identification of only a single gene. The recent release of first-draft genome sequencing data for R. microplus (Bellgard et al., 2012), together with genome data already available for Ixodes scapularis (Hill and Wikel, 2005; van Zee et al., 2007), has stimulated gene discovery in these and other ticks. However, these sequencing data sets need to undergo additional analyses before useful information may be extracted. Additionally, bioinformatic analyses and results should be confirmed by biological evidence such as protein characterisation and functional studies.

Previous EST studies showed lineage-specific expansion of several predicted secretory protein families for both hard and soft ticks (Mans et al., 2002b, 2008a; Mans and Neitz, 2004). These studies pointed to a series of gene duplication events after the divergence of the Ixodidae and Argasidae families. Such duplications provide the genetic variance that enables phenotypic hypervariability and may result in many paralogues with slightly altered specificities that can target a range of host and native proteins (Ohta, 1994, 2000; Christeller, 2005). The predominant predicted secretory protein families, i.e. lipocalins, basic tail secretory protein, Kunitz domaincontaining peptides and metalloproteases are common between hard and soft ticks. Of these, the Kunitz domain-containing protein family alone accounts for between $5 \%$ and $20 \%$ of all predicted 
secretory proteins (Ribeiro et al., 2006; Chmelar̆ et al., 2008; Francischetti et al., 2008a,b; Mans et al., 2008a). In I. scapularis, arguably the most intensively studied tick in North America due to its vector capacity for Lyme disease, the Kunitz domain-containing protein family comprises $10 \%$ of all predicted secretory proteins (Ribeiro et al., 2006). Even though several Kunitz proteins have been identified and/or characterised in R. microplus (Tanaka et al., 1999; de Miranda Santos et al., 2004; Sasaki et al., 2004; Rachinsky et al., 2007; Macedo-Ribeiro et al., 2008), the larger Kunitz domain-containing protein family has not been specifically studied within this tick species.

Kunitz-type proteins also have significant roles within other parasites. In the black fly, Simulium vittatum, they have been shown to inhibit coagulation and have a possible role in modulating inflammatory responses (Tsujimoto et al., 2012). In the flatworm, Echinococcus granulosus, a family of Kunitz domain-containing proteins have been implicated in the initial stages of infection (González et al., 2009), while many other parasites have recruited the Kunitz-scaffold into their venom proteins (reviewed by Fry et al. (2009)). In hookworms such as Ancylostoma ceylanicum it has been shown that Kunitz-type inhibitors protect the parasite against digestive serine proteases present in the host's small intestine (Costa et al., 2009; Tsujimoto et al., 2012). Immunisation against the $A$. ceylanicum Kunitz-type inhibitors confers partial protection against hookworm-associated growth delay, lending support for their possible use as parasite vaccine candidates (Chu et al., 2004).

The Kunitz protein family members, of which bovine pancreatic trypsin inhibitor (BPTI) is the type member (Burck et al., 1967; Cerwinsky et al., 1967), are typically serine protease inhibitors. One of their crucial functions in vertebrates is the control of haemostasis. Human tissue factor pathway inhibitor (TFPI) is a potent anti-coagulant that achieves inhibition of essential serine proteases of the blood coagulation cascade, such as factor Xa (fXa) and factor VIIa (fVIIa), through its three Kunitz domains (Lwaleed and Bass, 2006). As blood coagulation is one of the primary and most robust defences of vertebrates against blood-feeding parasites, it is unsurprising that all ticks secrete an assortment of anti-haemostatic proteins (reviewed by Maritz-Olivier et al. (2007) and 
Corral-Rodríguez et al. (2009)). These anti-coagulants serve to keep blood in its fluid form in both the feeding lesion and the tick's midgut, enabling the parasite to both obtain and digest its blood meal.

Various tick-derived Kunitz proteins that inhibit the serine proteases of the blood coagulation cascade, as well as soft tick-derived platelet aggregation inhibitors, have been identified. Ornithodorin and savignin are both bi-Kunitz thrombin inhibitors isolated from the soft ticks, Ornithodoros moubata (van de Locht et al., 1996) and Ornithodoros savignyi (Mans et al., 2002a). Despite having similarity to BPTI, they do not bind to thrombin via the Kunitz substratebinding loop, but instead insert their $\mathrm{N}$ - and C-terminal residues into thrombin's active site cleft and basic fibrinogen recognition exosite, respectively. Similar binding modes have been inferred for the hard tick-derived thrombin inhibitors, boophilin from R. microplus (Macedo-Ribeiro et al., 2008) and hemalin from Haemaphysalis longicornis (Liao et al., 2009), as well as for the soft tick-derived fX(a) inhibitors, tick anticoagulant protein from O. moubata (Wei et al., 1998) and fXa inhibitor (fXaI) from O. savignyi (Joubert et al., 1998). Multi-Kunitz inhibitors of fX(a) have also been isolated from the hard tick I. scapularis (Francischetti et al., 2002, 2004). Platelet aggregation inhibitors from soft ticks that use the Kunitz domain substrate-binding loop to present an RGD integrin recognition motif have also been characterised. Savignygrin isolated from O. savignyi is the most well-known of these (Mans et al., 2002c), but several savignygrin homologues have been identified in other soft ticks, including monogrin in Argas monolakensis (Mans et al., 2008b), disagregin in O. moubata (even though it presents RED instead of RGD (Karczewski et al., 1994; Karczewski and Connolly, 1997)) and RGD-presenting Kunitz proteins have also been found in Ornithodoros parkeri (Francischetti et al., 2008a).

Two TFPI homologues, ixolaris and penthalaris, have been identified and characterised in $I$. scapularis and have been shown to be potent anti-coagulants (Francischetti et al., 2002, 2004; Monteiro et al., 2005, 2008; Nazareth et al., 2006). They share sequence homology with TFPI and have anti-coagulant capabilities within the nanomolar range. In the case of ixolaris, this is achieved 
through inhibition of the tissue factor/fVIIa complex by using fX(a) as a scaffold (Francischetti et al., 2002). Penthalaris and ixolaris also form part of the larger Kunitz domain-containing protein family in I. scapularis (Ribeiro et al., 2006) which has developed through lineage-specific expansion.

Lineage-specific expansion of the Kunitz protein family has been previously observed in all studied ticks (Valenzuela et al., 2002; Francischetti et al., 2005, 2008a,b; Ribeiro et al., 2006; Alarcon-Chaidez et al., 2007; Batista et al., 2008; Chmelar̆ et al., 2008; Mans et al., 2008a; Dai et al., 2012). Previous studies have shown that this protein family has evolved through gene duplication within the main tick families but after the divergence of hard and soft ticks (Mans et al., 2002b, 2008a,b; Mans and Neitz, 2004). The Kunitz protein family has therefore expanded in response to the blood-feeding environment that the Ixodidae and Argasidae encountered individually. Recently, Dai et al. (2012) showed that a greater expansion of the Kunitz-type proteins within hard ticks, specifically I. scapularis, likely facilitates the long-term blood-feeding behaviour of these ticks. It is likely that a similar, expanded Kunitz domain-containing protein family is present in R. microplus, with the members showing homology to the TFPI-like proteins, ixolaris and penthalaris.

Due to a lack of knowledge regarding a TFPI-like Kunitz protein family in the economically important $R$. microplus, we undertook to identify TFPI-like Kunitz domain-containing proteins in the cattle tick. Our preliminary identification of this protein family, using available $R$. microplus sequence databases, revealed a family of 42 homologues. Phylogenetic analysis uncovered both ancient and recent gene- and domain duplications, supporting previous observations that lineagespecific expansions of the Kunitz protein family occurred in all studied ticks. More exhaustive studies on this family of proteins will be of great significance once the complete genome of the cattle tick becomes available. This will allow intensive cross-genera analyses, facilitating a deeper insight into the evolution of ticks and their independent adaptation to a blood-feeding lifestyle. 


\section{Materials and methods}

\subsection{Identification of TFPI-like Kunitz domain-containing proteins in R. microplus}

In order to assemble a data set of TFPI-like Kunitz domain-containing homologues, the Basic Local Alignment Search Tool (BLAST) (Altschul et al., 1997) was used to identify sequences within available $R$. microplus sequence databases that had similarity with the amino acid sequences of BPTI, TFPI, ixolaris and penthalaris (Table 1). BLAST searches were performed against a contig database (BmiContig compiled by C. Maritz-Olivier) assembled from the R. microplus gene index (BmiGi) (available at the Dana-Farber Cancer Gene Index Project, DFCI, at http://compbio.dfci.harvard.edu/tgi/tgipage.html), as well as against $R$. microplus sequences available from the National Centre for Biotechnology Information (NCBI, available at http://www.ncbi.nlm.nih.gov). Sequences that showed significant similarities (E-value $\leq 5 \times 10^{-2}$ ) to at least two of the four query sequences were included in further analyses. The correct reading frame for nucleotide sequences retrieved during BLAST searches was determined by aligning the various translated open reading frames with the amino acid sequence of the TFPI protein.

\subsection{Bioinformatic analysis of homologues}

The assembled sequences were submitted for domain-, signal peptide- and transmembrane region-prediction to online bioinformatic tools available through the ExPASy molecular biology server (available online at http://www.expasy.ch). Domain prediction was performed using both SMART version 6 (Simple Modular Architecture Research Tool, (Schultz et al., 1998; Letunic et al., 2008)) and Pfam version 24.0 (Finn et al., 2010) software. Signal peptide and transmembrane regions were predicted by SignalP version 3.0 (Nielsen et al., 1997; Bendtsen et al., 2004) and TMHMM version 2.0 (Kahsay et al., 2005) software, respectively. Sequences containing premature stop codons were discarded, except in the case of one (TC1750pt) where it was apparent that such a premature stop codon was the result of a nonsense mutation within the Kunitz domain. Remaining 
sequences were curated by truncation to the starting methionine, if one was apparent, and by discarding parts of the sequence that were separated from predicted Kunitz domains with stop codons. Forty-two TFPI-like Kunitz domain-containing homologues were identified in this manner.

\subsection{Phylogenetic analysis of complete homologue sequences}

The full amino acid sequences of all the homologues were aligned to the four initial query sequences using the ClustalX program, version 2.0.7 (Larkin et al., 2007) and phylogenetic trees were inferred from the alignment with the use of the MEGA4 program, build\# 4028 (Tamura et al., 2007). Visualisation of the underlying relationships between the various sequences was achieved by employing the maximum parsimony (MP), minimum evolution (ME), neighbor-joining (NJ) and unweighted pair group mean average (UPGMA) methods. The Poisson correction model for amino acid substitution was employed and, due to varying sequence lengths of homologues, all positions that contained missing data or alignment gaps were eliminated only in pairwise sequence comparisons. The reliability of the generated phylogenetic trees was evaluated by means of 10,000 bootstrap replicates. For all other analysis options the program default settings were used. The correct topology and analysis method was accepted as the method that yielded a tree that had the greatest number of nodes with fair support (present in at least $50 \%$ of bootstrap replicates).

\subsection{Phylogenetic analysis of individual Kunitz domains from homologues}

The 42 homologues were divided into their individual core Kunitz domains by truncating the domains at positions 4 and 56 (BPTI numbering according to Schechter and Berger (1967)). Partial Kunitz domains were not included. To be able to infer divergence times of the $R$. microplus Kunitz protein family and to show its lineage-specific expansion, an outgroup of Argasidae single Kunitz domain-containing sequences was selected and processed in the same manner and included in the analysis. These were savignygrin from O. savignyi, monogrin 1A and 1B from Argas monolakensis and two savignygrin-like transcripts from $O$. parkeri herein referred to as 
ORNIPARK9 and ORNIPARK10 (Table 1). The final data set contained 98 sequences of which 93 were from homologues retrieved from online $R$. microplus databases.

Alignment of codons within the various Kunitz domains was manually refined by using the amino acid sequence alignments as guides. Phylogenetic analyses were performed using Bayesian Evolutionary Analysis by Sampling Trees (BEAST) version 1.5.3 (Drummond and Rambaut, 2007). Three taxon sets with normal prior distributions were calibrated with divergence times from Jeyaprakash and Hoy (2009). These were Ixodida, which included all sequences $(300 \pm 27$ million years ago (MYA)), Argasidae containing the five soft tick sequences $(214 \pm 28$ MYA) and $93 R$. microplus sequences representing the Metastriate taxon set (134 \pm 22 MYA). The General Time Reversible (GTR) substitution model was employed, base frequencies were estimated and the site heterogeneity model "Gamma + Invariant Sites" with four gamma categories was selected. Two other models were also evaluated, one using the SRD06 model and another partitioning the codons separately, but the best fitting model was selected as one that correctly resolved the outgroup. A relaxed uncorrelated lognormal clock was used with the Yule tree prior for speciation and a starting tree was generated with the UPGMA method. Ten simulation runs, each with a Markov Chain Monte Carlo (MCMC) chain length of 10,000,000, were independently performed with parameter logging at every $1,000^{\text {th }}$ generation. Result files from independent analyses were analysed using Tracer (version 1.5.0) and software tools included in the BEAST package. The resultant tree was visualised with FigTree, version 1.3.1.

In order to obtain bootstrap support for the Bayesian tree, phylogenies were also generated using MEGA4. All methods (MP, ME, NJ and UPGMA) were employed with complete deletion of missing data or alignment gaps and inclusion of all codon positions $\left(1^{\text {st }}+2^{\text {nd }}+3^{\text {rd }}+\right.$ non-coding $)$. The nucleotide substitution model was the Maximum Composite Likelihood (MCL) and 1,000 bootstrap replicates were performed to infer confidence. The validity of the trees were evaluated against the Bayesian tree, and the tree with the highest number of nodes supported by either the 
posterior probability from BEAST and/or the bootstrap support from MEGA4 was selected as the most appropriate. This was the tree recovered by the ME method.

\subsection{Analysis of family expansion as a result of domain duplication}

To ascertain whether duplication events occurred that allowed expansion of the TFPI-like Kunitz domain-containing protein family in $R$. microplus, we analysed the level of similarity and identity within several clades identified during phylogenetic analysis of the individual Kunitz domains. We defined ancient duplications as sequences that have an amino acid identity of $40 \%$ or higher and a nucleotide identity of at least $50 \%$. Recent duplications were defined as those with an amino acid identity of no less than $85 \%$ and a minimum nucleotide identity of $90 \%$. The data set comprised of sub-clades containing at least three sequences, with a maximum divergence time of 55 MYA between the sequences.

\section{Results}

\subsection{Identification of TFPI-like Kunitz proteins in R. microplus and bioinformatic analysis}

Forty-two sequences with significant similarity (E-value $\leq 5 \times 10^{-2}$ ) to at least two query sequences (BPTI, TFPI, ixolaris and penthalaris) were identified during BLAST searches of $R$. microplus sequences in the BmiContig and NCBI databases. Analyses of these sequences revealed the presence of at least one Kunitz domain per sequence, up to a maximum of nine Kunitz domains (as for TC4684), with an average of 2.3 Kunitz domains per sequence (Fig. 1). Only three of the retrieved homologues were annotated in NCBI, while remaining sequences were of unknown affinity. EU636772 was described as a putative chymotrypsin inhibitor precursor (GenBank accession number EU636772, (Lima et al., 2010)), TC4205 corresponded to the two boophilin isoforms (G2 and H2, GenBank accession numbers $\underline{\mathbf{1 7 5 2 9 5 6 4}}$ and $\underline{\mathbf{1 7 5 2 9 5 6 6}}$, respectively) and 
TC6750 was described as a putative secreted protein known as penthaplus (GenBank accession number ABA55034.1).

The Bilaris family was the predominant group, constituting almost half of all Kunitz domain-containing proteins (17 sequences, $44 \%$ of the total), followed by the Monolaris and Trilaris families consisting of 14 sequences (33\%) and five sequences (14\%), respectively (Figs. 2 4). However, five of the 42 sequences contained partial Kunitz domains predicted at the N-terminal (CK192299, CV441191, TC4684, TC6491, TC13756) and others did not present with an initial methionine.

Approximately $40 \%$ of the full Kunitz domains presented a lysine or arginine in the P1 position, similar to BPTI and TFPI. The alignment of the individual Kunitz domain amino acid sequences is shown in Supplementary Fig. S1. Signal peptides were predicted for 17 sequences (41\%) with 24 remaining non-secretory $(60 \%)$. Only a single sequence had a predicted signal anchor (TC334). Twelve of the 17 secretory sequences (71\%) were contained within the Bilaris group. Furthermore, transmembrane regions were predicted for four of the 42 sequences (TC334, TC2049, TC6502 and TC8213), amounting to $10 \%$ of the total. The signal peptide predicted for TC8213 spanned most of the transmembrane region predicted for this transcript. In the case of TC334 the predicted signal anchor also spanned part of the transmembrane region.

Upon considering the alignment of the TFPI-like Kunitz proteins (Figs. 2 - 4), the disulphide-bonded cysteine residues of the Kunitz domain remained the most conserved residues, with partial conservation only for the second and fourth cysteines (Figs. $3-4$ ). This loss of conservation of $\mathrm{C}_{2}$ and $\mathrm{C}_{4}$ was also present in two of the initial query sequences, ixolaris and penthalaris, which both originate from the tick, I. scapularis.

\subsection{Phylogenetic analyses of complete amino acid sequences}

Phylogenetic analyses of full-length amino acid sequences using the MEGA4 program revealed that the NJ method yielded a consensus tree (Fig. 5) with the greatest degree of support. 
Of the 43 nodes, 28 displayed fair support (present in at least $50 \%$ of bootstrap replicates) and 16 of these had good support (present in at least $75 \%$ of bootstrap replicates).

The homologues were grouped into four major clades (Clades I - IV) with 16, nine, four and 13 homologue sequences, respectively (Fig. 5). Clade I was the largest and most diverse clade, containing sequences from every multi-Kunitz family as well as two of the initial query sequences, namely TFPI and penthalaris. The sub-clade, Clade Ia, had good support for all nodes. Both ixolaris and BPTI grouped into Clade II, which was composed almost exclusively of Monolaris sequences, with the exception of three Bilaris sequences (ixolaris, TC3418, TC5548). The smallest of the major clades was Clade III, which contained three Bilaris and one Monolaris sequence, and which was basal to both Clades I and II. Clade IV consisted mainly of Bilaris sequences but also contained a Monolaris sub-assemblage (Clade IVa), as well as three Trilaris and one penthalaris sequence scattered within Clade IVc. The Monolaris sub-clade (Clade IVa) was located basal to the rest of the sub-clades in Clade IV.

\subsection{Phylogenetic analysis of individual Kunitz domains}

An outgroup of five soft tick sequences was used to study the relationships between the individual Kunitz domains from the homologues (Fig. 6). The alignment of the individual Kunitz domain nucleotide sequences is shown in Supplementary Fig. S2. Three taxon sets were defined and used to calibrate the phylogenetic tree and the recovered divergence times of the sets, together with the lower and upper 95\% highest posterior density intervals (HPDs), are given in Table 2. Bayesian and ME analysis showed that the 93 Kunitz domains grouped into two major clades (Clades A and B) and one minor clade (Clade V), while the five soft tick sequences formed a basal outgroup to the $R$. microplus sequences. The major clades could each be subdivided into two minor clades (Clade A: Clades I and II; Clade B: Clades III and IV) resulting in a total of five minor clades. Fifty-one of the 96 nodes (53\%) had posterior probability support, while 33 nodes had bootstrap support (34\%). 


\subsection{Evidence for domain duplications as a method of family expansion}

We analysed the phylogenetic tree of the individual Kunitz domains for evidence of domain duplication. By focusing only on clades containing at least three individual Kunitz domains and a maximum divergence time of 55 MYA, we identified nine sub-clades with evidence for both recent and ancient domain duplication (Fig. 6 and Table 3). All of the Kunitz domains that formed part of the nine sub-clades, except for CV450668K3 in Clade IIIb, were duplications of the other Kunitz domains within their respective sub-clade. The sub-clade sizes ranged from 15 Kunitz domains within Clade IIc to three sub-clades containing only three domains (Clade Ia, Clade IIIa, Clade IIIc).

The 15 Kunitz domains that formed Clade IIc belonged to only seven transcripts, and nine were Kunitz domains from transcripts TC1750 and TC4684. The Kunitz domains of these two homologues were spread over Clades I and II, but Clade I only contained a single domain from each transcript (TC1750K2 and TC4683K3) (Fig. 7). The Kunitz domains present in Clade II could be divided into three groups: TC1750K7 + TC4684K9, TC1750K1 + TC4684K2, with the third group containing the rest of the sequences. When the complete sequences of TC1750 and TC4684 were aligned their overall amino acid identity and similarity were $58 \%$ and $66 \%$, respectively (Fig. 8).

\section{Discussion}

We set out to determine whether a family of TFPI-like Kunitz domain-containing proteins is present within $R$. microplus, and whether such a family had evolved through lineage-specific expansion of ancestral Kunitz domains. We successfully identified a TFPI-like Kunitz domaincontaining protein family comprising 42 proteins that evolved through both ancient and recent domain- and gene duplication. This correlates with previous hard and soft tick studies in which similar expansions of secretory protein families, including Kunitz protein families, were observed. These lineage-specific expansions likely occurred during individual adaptations of hard and soft 
ticks to blood-feeding behaviour after their divergence from the ancestral tick (Mans et al., 2002b, 2008a; Mans and Neitz, 2004). It is apparent that the Kunitz protein families are of integral importance to the haematophagous lifestyle of both hard and soft ticks.

Several previously identified tick Kunitz proteins have been shown to have anti-haemostatic functions, but despite their similarity to both TFPI and BPTI most have been shown to inhibit their targets through novel and non-canonical binding methods (Corral-Rodríguez et al., 2009). In some cases, loss of the $\mathrm{C}_{2}-\mathrm{C}_{4}$ disulphide bond is thought to further facilitate unconventional binding manners (Demchenko, 2001). Furthermore, tick-derived Kunitz proteins that inhibit novel and unexpected targets have been described. In the family of TFPI-like Kunitz proteins we identified during this study, we noted both losses of the cysteines responsible for the $\mathrm{C}_{2}-\mathrm{C}_{4}$ disulphide bridge as well as presentation of $\mathrm{P}_{1}$ residues that were not expected to facilitate canonical inhibition of vital proteases of the blood coagulation cascade. This indicates that the TFPI-like Kunitz domaincontaining proteins of $R$. microplus likely bind to their targets through novel ways and/or cause inhibition of unconventional targets. This family could therefore be a rich source of novel Kunitztype inhibitors with diverse and unexpected targets.

One of the aims of this study was to identify TFPI homologues in $R$. microplus that may inhibit blood coagulation, similar to the anti-haemostatics, ixolaris and penthalaris, that were found in I. scapularis (Francischetti et al., 2002, 2004). We discerned a family of 42 of these proteins, which was comprised primarily of Kunitz domain-containing proteins belonging to the Monolaris and Bilaris groups, with the Bilaris group predominating. This distribution of Kunitz proteins is similar to that already reported for ticks where the Monolaris and Bilaris groups are the dominant groups. Which group predominates differs among species, for example in the hard tick $I$. scapularis the Monolaris group is approximately twice the size of the Bilaris group and this lineagespecific expansion has been shown to impart long-term blood feeding functionality (Dai et al., 2012). Proteins containing three or more Kunitz domains have to date only been observed in hard ticks. In fact, the observation of a Nonalaris transcript within ticks (TC4684) is the first to our 
knowledge. The Heptalaris transcript present in this study (TC1750) had previously been described by Rachinsky et al. (2007) (identified as TC9311) who suggested that due to variable splicing it could give rise to several shorter Kunitz domain-containing proteins. Whether this is also the case for TC4684, a close homologue of TC1750, should be confirmed through additional proteomic studies. Many of the transcripts were truncated, which prevented complete sequence annotation. Nonetheless, several transcripts possessed predicted signal peptides. This supports the expected function of these proteins, namely that anti-coagulant Kunitz proteins would need to localise in the extracellular matrix so that they may be secreted into the feeding lesion or tick gut where maintenance of anti-haemostasis is required.

When the phylogeny of the TFPI-like Kunitz domain-containing proteins is considered, it is evident that the protein sequences did not group strongly according to the number of Kunitz domains they possess. Instead, all of the major clades are comprised of sequences belonging to at least two of the Kunitz families (Clades I to IV, Fig. 5) with Monolaris-type sequences generally in a basal position to the various clades. The phylogeny also showed some indications of gene duplication events, such as Monolaris sequences and sub-clades that were basal to multi-Kunitz sequences, as well as sequence groups in each major clade with strong support and short branch lengths, indicating little divergence from their most recent common ancestor (MRCA). Furthermore, the phylogeny of the individual Kunitz domains formed two major clades (Clades A and B, Fig. 6) that comprised almost all of the Kunitz domains from multi-domain transcripts. When the two phylogenies were compared, it became apparent that, except for seven transcripts (CK192837, TC185, TC2049, TC3418, TC5548, TC8213 and TC13756), all of the multi-Kunitz transcripts within Clade I have their Kunitz domains within Clade A. Similarly, multi-Kunitz transcripts residing in Clade IV exclusively grouped within Clade B. We therefore propose that the common ancestor of Clades A and B was likely a Monolaris-type transcript that underwent gene duplication and subsequent expansion to give rise to the two major families of Kunitz domaincontaining proteins within $R$. microplus. 
The estimated divergence times of the Ixodida, Argasidae and Metastriate lineages, as calculated in this paper by the phylogeny of the individual Kunitz domains, corresponded well with those presented by Jeyaprakash and Hoy (2009). In all cases there was an overlap between the $95 \%$ confidence intervals of the divergence times and in the case of the Argasidae the mean divergence time calculated in the present study (214 MYA) corresponded precisely with that previously estimated by Jeyaprakash and Hoy (2009). The mean haploid genome size of $R$. microplus ( 7.1 Gbp) has been reported as approximately double that of the rest of the Ixodidae ( 2.67 Gbp) (Ullmann et al., 2005; Geraci et al., 2007). This difference in size could be indicative of a whole genome duplication event in this tick. The MRCA of the two major Kunitz domain clades that we observed was estimated to have occurred at 150 MYA and the subsequent divergence of these clades could correspond to such a genome duplication event. Therefore, we propose that the ancient whole genome duplication that is expected to have occurred within $R$. microplus took place at least 150 MYA.

Further analysis of the sub-clades within the phylogeny of the individual Kunitz domains revealed evidence for both ancient and recent gene and domain duplications. We observed several recent gene duplications and all of these had divergence times of less than 11.4 MYA, with the most recent divergence only 1.05 MYA. The most convincing evidence for duplication, manifesting both gene and domain duplications, was in the cases of TC1750 and TC4684. A potential evolutionary pathway for these transcripts is discussed below and shown in Fig. 9.

The evolutionary path of the TC1750 and TC4684 homologues within R. microplus potentially started with the duplication of a single ancestral Kunitz domain belonging to a Monolaris-type protein (Fig. 9). One of the domains could subsequently have become the ancestor of TC1750K2, TC4684K1 and TC4684K3 (we will call this the primary domain - Kunitz domains from Clade I, Fig. 6), while the other became the ancestor of all the other Kunitz domains within TC1750 and TC4684 (the secondary domain - Kunitz domains from Clade II, Fig. 6). A series of domain duplication events then probably followed, during which five additional copies of the 
secondary domain were produced. At this stage, the MRCA of TC1750 and TC4684 must have existed, namely a 7-Kunitz protein comprising of a single primary Kunitz domain and six secondary Kunitz domains.

After the initial evolutionary processes involving domain duplications and expansions, a single gene duplication must have followed to allow divergence of TC1750 and TC4684. TC1750 probably continued to diverge without any further intra-transcript domain duplications, resulting in seven Kunitz domains and placing it in the Heptalaris group of Kunitz proteins. It seems that one domain (TC1750K6) could have been duplicated from TC1750 to give rise to a new, separate Kunitz protein, namely TC1750pt. This gene consists of a single Kunitz domain, TC1750ptK1, but a single nonsense mutation resulted in the appearance of a premature stop codon (Tyr $\rightarrow$ Stop).

TC4684 must have undergone a minimum of two further domain duplications to result in its current form consisting of at least nine Kunitz domains (Nonalaris group). Conceivably, one domain duplication produced TC4684K1 (primary domain type) and another produced TC4684K7 (secondary domain type). As TC4684 has an initial truncated Kunitz domain it is possible that it possesses additional Kunitz domains. Despite the inferred gene duplication event allowing TC1750 and TC4684 to diverge from each other, they still retain a high degree of amino acid identity (58\%) and similarity $(66 \%)$.

When only a single gene copy is present, it is subject to purifying selection that prevents the accumulation of deleterious mutations (Ohno, 1970). However, as soon as gene duplication occurs, both homologues experience relaxed selection constraints that allow them to enter a period of accelerated evolution (Lynch and Katju, 2004). This could eventually result in novel functionality, whether through different temporal or spatial expression, the acquisition of additional targets or neofunctionalisation. Establishment of inhibitor variability is often associated with gene and domain duplication (Hill and Hastie, 1987; Christeller, 2005). Thus, it is common for small inhibitor families to contain individual members with varied specificities. It is likely that the $R$. microplus TFPI-like Kunitz protein family has undergone expansion so that the family may inhibit 
a diverse group of targets. This is further supported by the observation that only approximately $40 \%$ of the full Kunitz domains analysed in this study have the typical $\mathrm{P}_{1}$ Lys or $\mathrm{P}_{1}$ Arg of BPTI and TFPI. These $\mathrm{P}_{1}$ residues are preferred by trypsin-like serine proteases such as those of the blood coagulation cascade (Helland et al., 1999).

In some domains of the TFPI-like Kunitz proteins (CK192299, CV450668, TC5548) there was a loss of the $\mathrm{C}_{2}-\mathrm{C}_{4}$ disulphide bridge. Loss of these cysteines has also occurred in Kunitz domains of ixolaris (K1) and penthalaris (K1, 2 and 5) (Francischetti et al., 2002, 2004). The absence of the $\mathrm{C}_{2}-\mathrm{C}_{4}$ bridge has been speculated to result in increased flexibility of the Kunitz domain binding loop, allowing interaction with sites other than the catalytic domain of the targeted serine protease (Demchenko, 2001). It could therefore be argued that these domains are more likely to bind novel targets. Nonetheless, tick-derived proteins that possess Kunitz domains with fully conserved cysteines have been shown to have novel and unexpected functions (Corral-Rodríguez et al., 2009). Considering all of the above, it is likely that inhibitors in this family could have both varied targets and/or novel manners in which they bind to both conventional and unconventional targets. The R. microplus TFPI-like Kunitz domain-containing protein family could therefore be a rich source of novel Kunitz-type inhibitors.

It is evident that the expansion of the TFPI-like Kunitz domain-containing protein family in R. microplus was facilitated through several gene and domain duplications, as well as a single genome duplication event. Such expansions are present in both hard and soft ticks but are lineagespecific as they occurred after the divergence of the major tick families. They therefore coincided with the independent adaptation of the hard and soft ticks to a blood-feeding environment (Mans et al., 2002b, 2008a; Mans and Neitz, 2004). Such adaptations would have required the evolution of proteins with novel functions that could facilitate blood-feeding behaviour and this was likely the evolutionary stimulus that resulted in the expansion of so many secretory protein families. As these expansions were initiated within the ancient tick lineages but have continued into recent times, it is 
clear that the expanded secretory protein families have always been and still are of integral importance to ticks.

While both hard and soft ticks show expansion of secretory protein families, the number of gene family members differ. Hard ticks possess more family members than the soft ticks (Mans et al., 2008a). The additional family members in hard ticks could have evolved in response to the long-term blood-feeding behaviour of the Ixodidae (Dai et al., 2012). These additional family members could assist during prolonged feeding times in several ways. Temporal variability in expression means that the various homologues could be expressed when needed during different times of the feeding cycle. In I. scapularis it has been observed that the expression of several Kunitz proteins are specifically intensified at $6 \mathrm{~h}$ or more post-attachment (Dai et al., 2012). This observation also specifically supports the importance of Kunitz proteins for long-term bloodfeeding within this ixodid tick. Through the expression of several functionally similar homologs that have variations in their amino acid sequences, the inhibitors can achieve a level of hypervariability, similar to the antigenic variance observed in many pathogens (Christeller, 2005). In the case of a parasite such as ticks, which employ these inhibitors to circumvent and disable host responses, such hypervariability would allow the inhibitors to evade the host's protective immune responses. Finally, expansion of the protein family enables the evolution of novel functions and the acquisition of new targets. The impressive arsenal of secretory proteins, including Kunitz-type inhibitors, that the tick possesses gives it the upper hand in the evolutionary arms race that host and parasite compete in.

Ticks may employ several mechanisms to achieve gene and domain duplication, and subsequently lineage-specific expansion of selected protein families. Support for polyploidisation within ticks, and specifically $R$. microplus, has been given by Geraci (2007) and Ullmann et al. (2005), although this is considered to have been an ancient event. It has also been estimated that close to $70 \%$ of the $R$. microplus genome consists of repetitive regions (Ullmann et al., 2005) which could cause duplications or deletions during unequal crossing over events. Reverse transcriptases 
have been described in several ticks (de la Fuente et al., 2007b; Chmelar̆ et al., 2008; Francischetti et al., 2008b) and recently both class I and II transposable elements have been found (Guerrero et al., 2010). Our team has also found retrotransposons flanking at least one of the Kunitz domaincontaining proteins identified in this study (C. Maritz-Olivier, personal communication). It is clear that the tick genome allows several avenues of exploitation to allow gene and domain duplication.

During the course of this study we identified an extensive family of TFPI-like Kunitz domain-containing proteins in $R$. microplus. The distribution of these is similar to that previously found in other ticks such as I. scapularis and approximately half of these homologues have predicted signal peptides and present $\mathrm{P}_{1}$ Lys or $\mathrm{P}_{1} \mathrm{Arg}$, supporting their expected role as secreted anti-haemostatic proteins. Nonetheless, unexpected and novel functions have often been described for tick-derived Kunitz proteins (Corral-Rodríguez et al., 2009) and further studies will be needed to confirm the anti-haemostatic functions of these proteins. We have found considerable evidence for gene and domain duplications within this family, including both ancient and recent duplications. We believe that this report represents the first comprehensive study of TFPI-like Kunitz proteins within $R$. microplus and expands our current knowledge of the evolution of Kunitz domaincontaining proteins within this tick. However, the homologues we identified were only those present within the NCBI and $R$. microplus EST databases (BmiGI). A first draft of the $R$. microplus genome sequence has recently been released (Bellgard et al., 2012) and once full coverage of the genome is available, an exhaustive analysis of all TFPI-like Kunitz domain-containing proteins within R. microplus would be possible.

\section{Acknowledgements}

This project was partially funded by the Wellcome Trust, United Kingdom, under the 'Animal Health in the Developing World' initiative through project 0757990 entitled 'Adapting recombinant anti-tick vaccines to livestock in Africa'. 


\section{References}

Alarcon-Chaidez, F.J., Sun, J., Wikel, S.K., 2007. Transcriptome analysis of the salivary glands of Dermacentor andersoni Stiles (Acari: Ixodidae). Insect Biochem. Mol. Biol. 37, 48 - 71.

Altschul, S.F., Madden, T.L., Schäffer, A.A., Zhang, J., Zhang, Z., Miller, W., Lipman, D.J., 1997. Gapped BLAST and PSI-BLAST: A new generation of protein database search programs. Nucl. Acids Res. 25, 3389-3402.

Batista, I.F.C., Chudzinski-Tavassi, A.M., Faria, F., Simons, S.M., Barros-Batestti, D.M., Labruna, M.B., Leão, L.I., Ho, P.L., Junqueira-de-Azevedo, I.L.M., 2008. Expressed sequence tags (ESTs) from the salivary glands of the tick Amblyomma cajennense (Acar: Ixodidae). Toxicon 51, $823-834$.

Bellgard, M.I., Moolhuijzen, P.M., Guerrero, F.D., Schibeci, D., Rodriguez-Valle, M., Peterson, D.G., Dowd, S.E., Barrero, R., Hunter, A., Miller, R.J., Lew-Tabor, A.E., 2012. CattleTickBase: An integrated Internet-based bioinformatics resource for Rhipicephalus (Boophilus) microplus. Int. J. Parasitol. 42, 161 - 169.

Bendtsen, J.D., Nielsen, H., von Heijne, G., Brunak, S., 2004. Improved prediction of signal peptides: SignalP 3.0. J. Mol. Biol. 340, 783 - 795.

Burck, P.J., Hamill, R.L., Cerwinsky, E.W., Grinnan, E.L., 1967. Acidic bovine pancreatic trypsin inhibitor. II. Specificity of inhibition. Biochemistry 6, 3180 - 3184.

Cerwinsky, E.W., Burck, P.J., Grinnan, E.L., 1967. Acidic bovine pancreatic trypsin inhibitor. I. Purification and physical characterization. Biochemistry 6, 3175 - 3179.

Chmelař, J., Anderson, J.M., Mu, J., Jochim, R.C., Valenzuela, J.G., Kopecký, J., 2008. Insight into the sialome of the castor bean tick, Ixodes ricinus. BMC Genom. 9, 233 - 253.

Christeller, J.T., 2005. Evolutionary mechanisms acting on proteinase inhibitor variability. FEBS J. $272,5710-5722$. 
Chu, D., Bungiro, R.D., Jr., Ibanez, M., Harrison, L.M., Campodonico, E., Jones, B.J., Mieszczanek, J., Kuzmic, P., Cappello, M., 2004. Molecular characterization of Ancylostoma ceylanicum Kunitz-type serine protease inhibitor: Evidence for a role in hookworm-associated growth delay. Infect. Immun. 72, 2214 - 2221.

Corral-Rodríguez, M.Á., Macedo-Ribeiro, S., Pereira, P.J.B., Fuentes-Prior, P., 2009. Tick-derived Kunitz-type inhibitors as antihemostatic factors. Insect Biochem. Mol. Biol. 39, 579-595.

Costa, A.F.D.V., Gasser, R.B., Dias, S.R.C., Rabelo, É.M.L., 2009. Male-enriched transcription of genes encoding ASPs and Kunitz-type protease inhibitors in Ancylostoma species. Mol. Cell. Probes 23, 298 - 303.

Dai, S.-X., Zhang, A.-D., Huang, J.-F., 2012. Evolution, expansion and expression of the Kunitz/BPTI gene family associated with long-term blood feeding in Ixodes scapularis. BMC Evol. Biol. 12, 4.

de la Fuente, J., Almazán, C., Canales, M., de la Lastra, J.M.P., Kocan, K.M., Willadsen, P., 2007a. A ten-year review of commercial vaccine performance for control of tick infestations on cattle. Anim. Health. Res. Rev. 8, 23-28.

de la Fuente, J., Blouin, E.F., Manzano-Roman, R., Naranjo, V., Almazán, C., de la Lastra, J.M.P., Zivkovic, Z., Jongejan, F., Kocan, K.M., 2007b. Functional genomic studies of tick cells in response to infection with the cattle pathogen, Anaplasma marginale. Genomics 90, 712722.

de Miranda Santos, I.K.F., Valenzuela, J.G., Ribeiro, J.M.C., de Castro, M., Costa, J.N., Costa, A.M., da Silva, E.R., Neto, O.B.R., Rocha, C., Daffre, S., Ferreira, B.R., da Silva, J.S., Szabó, M.P., Bechara, G.H., 2004. Gene discovery in Boophilus microplus, the cattle tick: The transcriptomes of ovaries, salivary glands and hemocytes. Ann. N. Y. Acad. Sci. 1026, $242-246$.

Demchenko, A.P., 2001. Recognition between flexible protein molecules: Induced and assisted folding. J. Mol. Recognit. 14, 42 - 61. 
Drummond, A.J., Rambaut, A., 2007. BEAST: Bayesian evolutionary analysis by sampling trees. BMC Evol. Biol. 7, 214.

Estrada-Peña, A., Sánchez Acedo, C., Quílez, J., Del Cacho, E., 2005. A retrospective study of climatic suitability for the tick Rhipicephalus (Boophilus) microplus in the Americas. Global Ecol. Biogeogr. 14, 565 - 573.

Finn, R.D., Mistry, J., Tate, J., Coggill, P., Heger, A., Pollington, J.E., Gavin, O.L., Gunesekaran, P., Ceric, G., Forslund, K., Holm, L., Sonnhammer, E.L., Eddy, S.R., Bateman, A., 2010. The Pfam protein families database. Nucl. Acids Res., D211 - D222.

Francischetti, I.M.B., Mans, B.J., Meng, Z., Gudderra, N., Veenstra, T.D., Pham, V.M., Ribeiro, J.M.C., 2008a. An insight into the sialome of the soft tick, Ornithodorus parkeri. Insect Biochem. Mol. Biol. 38, 1-21.

Francischetti, I.M.B., Mather, T.N., Ribeiro, J.M.C., 2004. Penthalaris, a novel recombinant fiveKunitz tissue factor pathway inhibitor (TFPI) from the salivary gland of the tick vector of Lyme disease, Ixodes scapularis. Thromb. Haemost. 91, 886 - 898.

Francischetti, I.M.B., Meng, Z., Mans, B.J., Gudderra, N., Hall, M., Veenstra, T.D., Pham, V.M., Kotsyfakis, M., Ribeiro, J.M.C., 2008b. An insight into the salivary transcriptome and proteome of the soft tick and vector of epizootic bovine abortion, Ornithodoros coriaceus. J. Proteomics 71, 493 - 512.

Francischetti, I.M.B., Pham, V.M., Mans, B.J., Andersen, J.F., Mather, T.N., Lane, R.S., Ribeiro, J.M.C., 2005. The transcriptome of the salivary glands of the female western black-legged tick Ixodes pacificus (Acari: Ixodidae). Insect Biochem. Mol. Biol. 35, 1142-1161.

Francischetti, I.M.B., Valenzuela, J.G., Andersen, J.F., Mather, T.N., Ribeiro, J.M.C., 2002. Ixolaris, a novel recombinant tissue factor pathway inhibitor (TFPI) from the salivary gland of the tick, Ixodes scapularis: Identification of factor X and factor Xa as scaffolds for the inhibition of factor VIIa/tissue factor complex. Blood 99, 3602-3612. 
Fry, B.G., Roelants, K., Champagne, D.E., Scheib, H., Tyndall, J.D.A., King, G.F., Nevalainen, T.J., Norman, J.A., Lewis, R.J., Norton, R.S., Renjifo, C., de la Vega, R.C.R., 2009. The toxicogenomic multiverse: Convergent recruitment of proteins into animal venoms. Annu. Rev. Genomics Hum. Genet. 10, 483 - 511.

Geraci, N.S., Johnston, J.S., Robinson, J.P., Wikel, S.K., Hill, C.A., 2007. Variation in genome size of argasid and ixodid ticks. Insect Biochem. Mol. Biol. 37, 399-408.

González, S., Fló, M., Margenat, M., Durán, R., González-Sapienza, G., Graña, M., Parkinson, J., Maizels, R.M., Salinas, G., Alvarez, B., Fernández, C., 2009. A family of diverse Kunitz inhibitors from Echinococcus granulosus potentially involved in host-parasite cross-talk. PLoS ONE 4, e7009.

Guerrero, F.D., Moolhuijzen, P., Peterson, D.G., Bidwell, S., Caler, E., Bellgard, M.I., Nene, V.M., Djikeng, A., 2010. Reassociation kinetics-based approach for partial genome sequencing of the cattle tick, Rhipicephalus (Boophilus) microplus. BMC Genom. 11, 374.

Helland, R., Otlewski, J., Sundheim, O., Dadlez, M., Smalås, A.O., 1999. The crystal structures of the complexes between bovine $\beta$-trypsin and ten P1 variants of BPTI. J. Mol. Biol. 287, 923942.

Hill, C.A., Wikel, S.K., 2005. The Ixodes scapularis Genome Project: an opportunity for advancing tick research. Trends Parasitol. 21, 151 - 153.

Hill, R.E., Hastie, N.D., 1987. Accelerated evolution in the reactive centre regions of serine protease inhibitors. Nature 326, 96 - 99.

Jeyaprakash, A., Hoy, M.A., 2009. First divergence time estimate of spiders, scorpions, mites and tick (subphylum: Chelicerata) inferred from mitochondrial phylogeny. Exp. Appl. Acarol. $47,1-18$.

Joubert, A.M., Louw, A.I., Joubert, F., Neitz, A.W.H., 1998. Cloning, nucleotide sequence and expression of the gene encoding factor $\mathrm{Xa}$ inhibitor from the salivary glands of the tick, Ornithodoros savignyi. Exp. Appl. Acarol. 22, 603-619. 
Kahsay, R.Y., Gao, G., Liao, L., 2005. An improved hidden Markov model for transmembrane protein detection and topology prediction and its applications to complete genomes. Bioinformatics 21, 1853 - 1858.

Karczewski, J., Connolly, T.M., 1997. The interaction of disagregin with platelet fibrinogen receptor, glycoprotein IIb-IIIa. Biochem. Biophys. Res. Commun. 241, 744-748.

Karczewski, J., Endris, R., Connolly, T.M., 1994. Disagregin is a fibrinogen receptor antagonist lacking the Arg-Gly-Asp sequence from the tick, Ornithodoros moubata. J. Biol. Chem. 269, 6702-6708.

Larkin, M.A., Blackshields, G., Brown, N.P., Chenna, R., McGettigan, P.A., McWilliam, H., Valentin, F., Wallace, I.M., Wilm, A., Lopez, R., Thompson, J.D., Gibson, T.J., Higgins, D.G., 2007. Clustal W and Clustal X version 2.0. Bioinformatics 23, 2947 - 2948.

Letunic, I., Doerks, T., Bork, P., 2008. SMART 6: Recent updates and new developments. Nucl. Acids Res. 37, D229 - D232.

Liao, M., Zhou, J., Gong, H., Bolbaatar, D., Shirafuji, R., Battur, B., Nishikawa, Y., Fujisaki, K., 2009. Hemalin, a thrombin inhibitor isolated from a midgut cDNA library from the hard tick Haemaphysalis longicornis. J. Insect Physiol. 55, 164 - 173.

Lima, C.A., Torquato, R.J.S., Sasaki, S.D., Justo, G.Z., Tanaka, A.S., 2010. Biochemical characterization of a Kunitz type inhibitor similar to dendrotoxins produced by Rhipicephalus (Boophilus) microplus (Acari: Ixodidae) hemocytes. Vet. Parasitol. 167, 279 287.

Lwaleed, B.A., Bass, P.S., 2006. Tissue factor pathway inhibitor: Structure, biology and involvement in disease. J. Pathol. 208, 327-339.

Lynch, M., Katju, V., 2004. The altered evolutionary trajectories of gene duplicates. Trends Genet. $20,544-549$.

Lynen, G., Zeman, P., Bakuname, C., Di Giulio, G., Mtui, P., Sanka, P., Jongejan, F., 2008. Shifts in the distributional ranges of Boophilus ticks in Tanzania: Evidence that a parapatric 
boundary between Boophilus microplus and B. decoloratus follows climate gradients. Exp. Appl. Acarol. 44, 147 - 164.

Macedo-Ribeiro, S., Almeida, C., Calisto, B.M., Friedrich, T., Mentele, R., Stürzebecher, J., Fuentes-Prior, P., Pereira, P.J.B., 2008. Isolation, cloning and structural characterisation of boophilin, a multifunctional Kunitz-type proteinase inhibitor from the cattle tick. PLoS ONE 3, e1624.

Mans, B.J., Andersen, J.F., Francischetti, I.M.B., Valenzuela, J.G., Schwan, T.G., Pham, V.M., Garfield, M.K., Hammer, C.H., Ribeiro, J.M.C., 2008a. Comparative sialomics between hard and soft ticks: Implications for the evolution of blood-feeding behavior. Insect Biochem. Mol. Biol. 38, 42-58.

Mans, B.J., Andersen, J.F., Schwan, T.G., Ribeiro, J.M.C., 2008b. Characterization of antihemostatic factors in the argasid, Argas monolakensis: Implications for the evolution of blood-feeding in the soft tick family. Insect Biochem. Mol. Biol. 38, 22-41.

Mans, B.J., Louw, A.I., Neitz, A.W.H., 2002a. Amino acid sequence and structure modeling of savignin, a thrombin inhibitor from the tick, Ornithodoros savignyi. Insect Biochem. Mol. Biol. 32, 821-828.

Mans, B.J., Louw, A.I., Neitz, A.W.H., 2002b. Evolution of hematophagy in ticks: Common origins for blood coagulation and platelet aggregation inhibitors from soft ticks of the genus Ornithodoros. Mol. Biol. Evol. 19, 1695 - 1705.

Mans, B.J., Louw, A.I., Neitz, A.W.H., 2002c. Savignygrin, a platelet aggregation inhibitor from the soft tick Ornithodoros savignyi, presents the RGD integrin recognition motif on the Kunitz-BPTI fold. J. Biol. Chem. 277, 21371-21378.

Mans, B.J., Neitz, A.W.H., 2004. Adaptation of ticks to a blood-feeding environment: Evolution from a functional perspective. Insect Biochem. Mol. Biol. 34, 1-17.

Maritz-Olivier, C., Stutzer, C., Jongejan, F., Neitz, A.W.H., Gaspar, A.R.M., 2007. Tick antihemostatics: Targets for future vaccines and therapeutics. Trends Parasitol. 23, 397-407. 
Monteiro, R.Q., Rezaie, A.R., Bae, J.S., Calvo, E., Andersen, J.F., Francischetti, I.M.B., 2008. Ixolaris binding to factor $\mathrm{X}$ reveals a precursor state of factor Xa heparin-binding exosite. Prot. Sci. 17, 1-8.

Monteiro, R.Q., Rezaie, A.R., Ribeiro, J.M.C., Francischetti, I.M.B., 2005. Ixolaris: A factor Xa heparin-binding exosite inhibitor. Biochem. J. 387, 871-877.

Nazareth, R.A., Tomaz, L.S., Ortiz-Costa, S., Atella, G.C., Ribeiro, J.M.C., Francischetti, I.M.B., Monteiro, R.Q., 2006. Antithrombotic properties of Ixolaris, a potent inhibitor of the extrinsic pathway of the coagulation cascade. J. Thromb. Haemost. 96, 7-13.

Nielsen, H., Engelbrecht, J., Brunak, S., von Heijne, G., 1997. Identification of prokaryotic and eukaryotic signal peptides and prediction of their cleavage sites. Prot. Eng. 10, 1 - 6.

Ohno, S., 1970. Evolution by gene duplication. Springer-Verlag, Berlin, Germany.

Ohta, T., 1994. On hypervariability at the reactive center of proteolytic enzymes and their inhibitors. J. Mol. Evol. 39, 614 - 619.

Ohta, T., 2000. Evolution of gene families. Gene 259, 45 - 52.

Peconick, A.P., Sossai, S., Girão, F.A., Rodrigues, M.Q.R.B., Souza e Silva, C.H., Guzman, F., Patarroyo, A.M., Vargas, M.I., Patarroyo, J.H., 2008. Synthetic vaccine (SBm7462) against the cattle tick Rhipicephalus (Boophilus) microplus: Preservation of immunogenic determinants in different strains from South America. Exp. Parasitol. 119, 37 - 43.

Rachinsky, A., Guerrero, F.D., Scoles, G.A., 2007. Differential protein expression in ovaries of uninfected and Babesia-infected southern cattle ticks, Rhipicephalus (Boophilus) microplus. Insect Biochem. Mol. Biol. 37, 1291-1308.

Ribeiro, J.M.C., Alarcon-Chaidez, F.J., Francischetti, I.M.B., Mans, B.J., Mather, T.N., Valenzuela, J.G., Wikel, S.K., 2006. An annotated catalog of salivary gland transcripts from Ixodes scapularis ticks. Insect Biochem. Mol. Biol. 36, 111-129.

Sasaki, S.D., Azzolini, S.S.A., Hirata, I.Y., Andreotti, R., Tanaka, A.S., 2004. Boophilus microplus tick larvae, a rich source of Kunitz type serine proteinase inhibitors. Biochimie 86, 643-649. 
Schechter, I., Berger, A., 1967. On the size of the active site in proteases. I. Papain. Biochem. Biophys. Res. Commun. 27, 157-162.

Schultz, J., Milpetz, F., Bork, P., Ponting, C.P., 1998. SMART, a simple modular architecture research tool: Identification of signaling domains. Proc. Natl. Acad. Sci. USA 95, 5857 5864.

Tamura, K., Dudley, J., Nei, M., Kumar, S., 2007. MEGA4: Molecular evolutionary genetics analysis (MEGA) software version 4.0. Mol. Biol. Evol. 24, 1596-1599.

Tanaka, A.S., Andreotti, R., Gomes, A., Torquato, R.J.S., Sampaio, M.U., Sampaio, C.A.M., 1999. A double headed serine proteinase inhibitor - human plasma kallikrein and elastase inhibitor - from Boophilus microplus larvae. Immunopharmacology 45, 171-177.

Tsujimoto, H., Kotsyfakis, M., Francischetti, I.M.B., Eum, J.H., Strand, M.R., Champagne, D.E., 2012. Simukunin from the salivary glands of the black fly Simulium vittatum inhibits enzymes that regulate clotting and inflammatory responses. PLoS ONE 7, e29964.

Ullmann, A.J., Lima, C.M.R., Guerrero, F.D., Piesman, J., Black, W.C., 2005. Genome size and organization in the black-legged tick, Ixodes scapularis, and the Southern cattle tick, Boophilus microplus. Insect Mol. Biol. 14, 217 - 222.

Valenzuela, J.G., Francischetti, I.M.B., Pham, V.M., Garfield, M.K., Mather, T.N., Ribeiro, J.M.C., 2002. Exploring the sialome of the tick Ixodes scapularis. J. Exp. Biol. 205, 2843-2864.

van de Locht, A., Stubbs, M.T., Bode, W., Friedrich, T., Bollschweiler, C., Höffken, W., Huber, R., 1996. The ornithodorin-thrombin crystal structure, a key to the TAP enigma? EMBO J. 15, 6011-6017.

van Zee, J.P., Geraci, N.S., Guerrero, F.D., Wikel, S.K., Stuart, J.J., Nene, V.M., Hill, C.A., 2007. Tick genomics: The Ixodes genome project and beyond. Int. J. Parasitol. 37, 1297-1305.

Wei, A., Alexander, R.S., Duke, J., Ross, H., Rosenfeld, S.A., Chang, C.H., 1998. Unexpected binding mode of tick anticoagulant peptide complexed to bovine factor Xa. J. Mol. Biol. $283,147-154$. 
White, N., Sutherst, R.W., Hall, N., Whish-Wilson, P., 2003. The vulnerability of the Australian beef industry to impacts of the cattle tick (Boophilus microplus) under climate change. Climatic Change 61, 157 - 190.

Willadsen, P., 2004. Anti-tick vaccines. Parasitology 129, S367-S387. 


\section{Legends to Figures}

Fig. 1. Distribution of Kunitz domain families in Rhipicephalus microplus. The Bilaris (Bi) and Monolaris (Mono) families are the predominant Kunitz domain families within the cattle tick, making up $41 \%$ and $33 \%$ of the total, respectively. The rest of the families (Tri-Kunitz to NonaKunitz) collectively make up less than one-third of the whole.

Fig. 2. Alignment of the Monolaris family in Rhipicephalus microplus. Fourteen of the 42 Kunitz domain-containing sequences retrieved during BLAST searches had a single predicted Kunitz domain, placing them in the Monolaris group. The query 'bovine pancreatic trypsin inhibitor' (BPTI, GenBank accession number 115114), which also has a single Kunitz domain, aligns with this group. The conserved cysteines involved in the disulphide bond formation and the bonds themselves are indicated. Bioinformatic prediction software showed that two of these have signal peptides, another has a signal anchor and two sequences have transmembrane regions. Black to grey shading represents conservation of amino acid similarity at each position with residues conserved between all sequences shaded in black. Sequence names are as obtained from the DFCI database, except for $\underline{\mathbf{E U 6 3 6 7 7 2}}$ which corresponds to a NCBI database entry.

Fig. 3. Alignment of the Bilaris family in Rhipicephalus microplus. Seventeen sequences had two predicted Kunitz domains, placing them in the Bilaris group. 'Ixolaris' (GenBank accession number $\underline{\mathbf{1 5 0 7 7 0 0 2}}$ ), from the Ixodes scapularis Bilaris family, is the query that aligns to this family. The conserved cysteines involved in the disulphide bond formation and the bonds themselves are indicated. Bioinformatic prediction software showed that 12 of these have signal peptides and two sequences have transmembrane regions. Black to grey shading represents conservation of amino acid similarity at each position with residues conserved between all sequences shaded in black. In the case of TC8213 the signal peptide region spans over the transmembrane region and therefore 
only the last four amino acids that are part of the transmembrane region are shown. Sequence names correspond to DFCI database entries.

Fig. 4. Alignment of the Trilaris and greater multilaris families in Rhipicephalus microplus. Eleven sequences had three or more predicted Kunitz domains, placing them in the groups ranging from the Trilaris to Nonalaris families. Seven of these have predicted signal peptides. Both 'tissue factor pathway inhibitor' (TFPI, GenBank accession number 125932) and 'penthalaris' (GenBank accession number $\underline{\mathbf{2 2 1 6 4 2 6 6}}$ ) are queries that align well with these families. The conserved cysteines involved in the disulphide bond formation and the bonds themselves are indicated. Black to grey shading represents conservation of amino acid similarity at each position with residues conserved between all sequences shaded in black.

Fig. 5. Phylogenetic analysis of tissue factor pathway inhibitor (TFPI)-like Kunitz proteins in Rhipicephalus microplus. A Neighbor-Joining analysis with 10,000 bootstrap replicates was performed on the four query sequences as well as the 42 homologues that were retrieved during BLAST searches. Nodes with open diamonds indicate $\geq 50 \%$ confidence while filled diamonds are for $\geq 75 \%$ confidence. The scale is the number of amino acid substitutions per site. Colored (shaded) circles show the number of Kunitz domains for each transcript and are as shown in Fig. 1. The open circle is included to show a node with a branch length that is less than 0.015 and difficult to discern.

Fig. 6. Phylogenetic analysis of individual Kunitz domains from homologues. The Bayesian Evolutionary Analysis by Sampling Trees (BEAST) generated tree for the individual complete Kunitz domains from the various homologues, as well as five soft tick sequences that form an outgroup, is shown. The 93 Rhipicephalus microplus Kunitz domains group into five minor clades (Clade I - V). Confidence support from the BEAST and Minimum Evolution (ME) tree is indicated 
with colored (shaded) circles: green (black) shows both posterior probability $(\geq 0.500)$ and bootstrap support ( $\geq 50 \%$ ); blue (dark grey) shows only posterior probability support for the branch; red (light grey) shows the branch has bootstrap support; open circles indicate that the branch is predicted by both the BEAST and the ME trees but does not have sufficient support from either. The scale is 50 million years ago (MYA) with the age of the ancestral node recovered as $255.3 \pm$ 0.9 MYA (95\% highest posterior density interval (HPD): 204.9, 310.2). Specific calibration points used as well as the divergence times recovered in this study are given in Table 2. The individual Kunitz domains were named by appending $\mathrm{K}$ and the Kunitz domain number within the homologue to the homologue's name, for example the third Kunitz domain of TC185 is named TC185K3.

Fig. 7. Alignment of individual Kunitz domains from the closely homologous multilaris sequences TC1750 and TC4684. When the individual complete Kunitz domains of TC1750 and TC4684 are aligned they form four groups. Only group A falls within Clade I while groups B - D form part of Clade II. This suggests that an ancient domain duplication resulted in the ancestors of the Kunitz domains present in Clades I and II, and those in Clade II underwent further duplications to give the current transcripts. Black to grey shading represents conservation of amino acid similarity at each position with residues conserved between all sequences shaded in black.

Fig. 8. Alignment of the closely homologous multilaris sequences TC1750 and TC4684. By aligning the full amino acid sequences of TC1750 and TC4684 it is clear that these two sequences evolved by gene duplication of a common ancestor. Furthermore TC4684 diverged from TC1750 by acquiring two more Kunitz domains through intra-domain duplication - these two domains were inserted before TC1750K1 and between TC1750K5 and TC1750K6. The overall amino acid identity between the two sequences is $58 \%$ with the amino acid similarity being $66 \%$. The conserved cysteines involved in the disulphide bond formation and the bonds themselves are 
indicated. Black to grey shading represents conservation of amino acid similarity at each position with residues conserved between all sequences shaded in black.

Fig. 9. Proposed evolutionary path for the closely homologous multilaris sequences TC1750 and TC4684. The evolutionary path resulting in TC1750 and TC4684 may be inferred from the phylogenetic relationship and sequence similarities of their individual Kunitz domains and overall sequence (Figs. $6-8$ ). Starting with an ancestral Kunitz domain, several intra-transcript domain duplications (dark red/dark grey arrows) were followed by a single gene duplication (bright red/light grey arrows), producing the most recent common ancestor (MRCA) of TC1750 and TC4684. TC1750 diverged further without additional domain duplications, while TC4684 gained two more Kunitz domains through duplications, resulting in these homologues having seven and nine Kunitz domains, respectively.

\section{Supplementary Figure legends}

Supplementary Fig. S1. Alignment of amino acid sequences of the individual Kunitz domains. The amino acid sequences of the 98 individual Kunitz domains were aligned. The cysteines involved in the disulphide bridge formation of the Kunitz fold remain the most conserved. The $\mathrm{P}_{1}$ residues are blocked in red.

Supplementary Fig. S2. Alignment of nucleotide sequences of the individual Kunitz domains. The nucleotide sequences of the 98 individual Kunitz domains were aligned manually to correspond to the amino acid alignment (Supplementary Fig. S1). The codons corresponding to the $\mathrm{P}_{1}$ residue are blocked in red. 
Figure

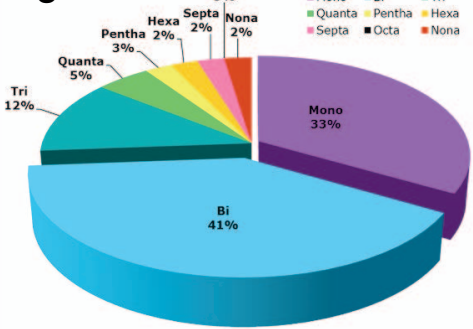




\section{Eigure $2-$ Signal Peptides Signal Anchor \\ - Transmembrane Regions \\ Disulphide Bonds}

\section{T027274 * 26}

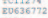
IID2:

Tesibopt cV4a1891 TC4754 to 3419 CV442296. To13715 тез34

TC21158

Tes 502

1010529

TC1212

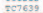

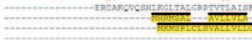

40

\begin{tabular}{|c|}
\hline 7021274 \\
\hline 80636772 \\
\hline sog: \\
\hline Te2150pt \\
\hline CV441191 \\
\hline ted 754 \\
\hline Te3419 \\
\hline CV442290 \\
\hline Tetis? \\
\hline tesse \\
\hline Te111s8 \\
\hline $\operatorname{Tc} 6502$ \\
\hline Tc1052s \\
\hline 201212 \\
\hline 703639 \\
\hline
\end{tabular}

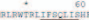

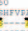

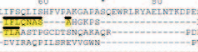
50

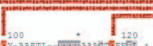

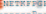

Y-AXקDS-

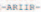

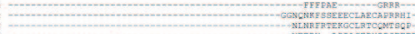

ances

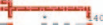

$\rightarrow \quad 360$

IIsc

200

- 228

240

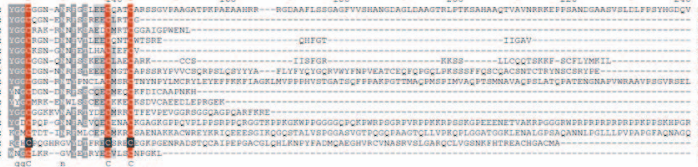




\section{-}

\section{0 \\ 1 ad}

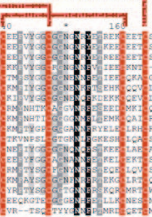

TruskKeEIAAASTVATELEHSATVA WIT3FFEFTAAASTVMTEDFH:ATVh-

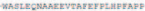
-APEKVE

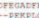
-PCKRPy ataris: IFImok CTREPK - Thespor

SSTWL TVICDVLAFGCLTKKNE DASF DNCW SRRMSA -NTSCPZ -INYRem -

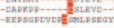
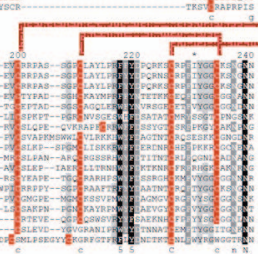

MIVAKESCR2INEY

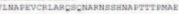

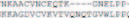

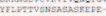

ADSSTY

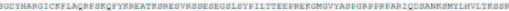

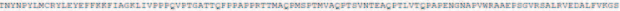




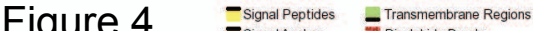
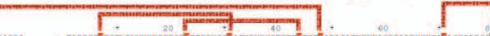

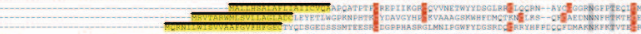

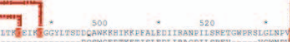

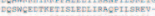

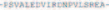
-vinersavisp

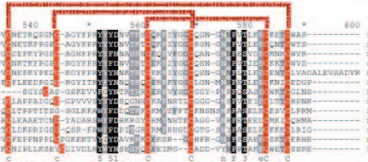

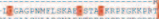

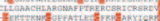

428

.

ess

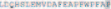

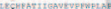
AYQDTOPISCAYEYPFWOA

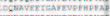

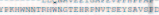

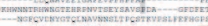

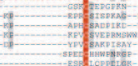

54 을 Thaveate

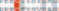
Pal Troves: Tivinged

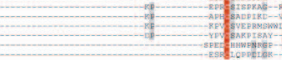

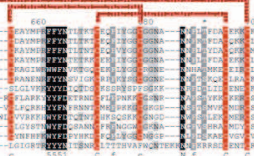


Figure 5

7 TC1750 - 9 TCA684

4 TC6491

2 TC5576

(3) TC7271

4 1C7639

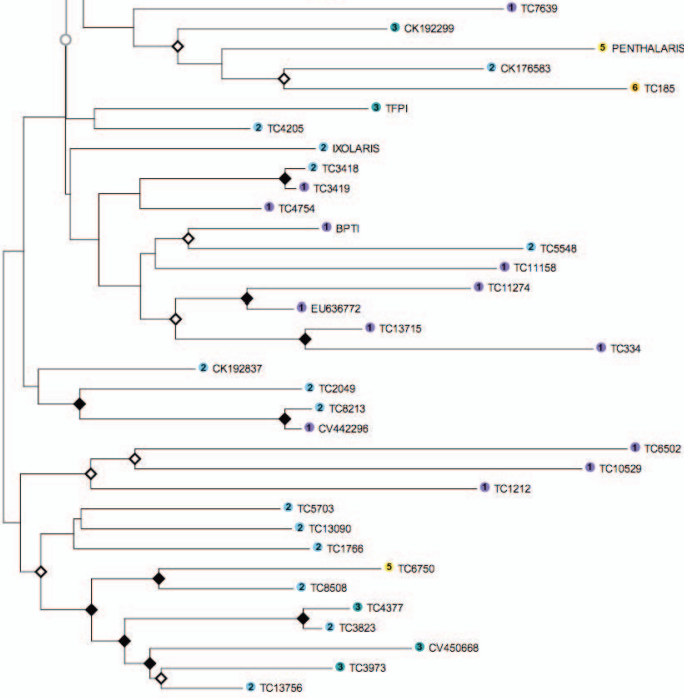

Clade Ilb

Clade II

Clade Ila

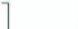

Clade Ic

Clade I

Clade Ib

.

Clade III

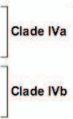

Clade IVc

Clade IV

0.2 
- Posterior probability $(\geq 0.500)$ and bootstrap ( $\geq 50 \%)$

- Posterior probability $(\geq 0.500)$

- Bootstrap ( $250 \%$ )

O Insufficient support

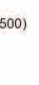

TC3519K1

TC8266K 1

TC12361K1

TC8492K2

- TC6492K1

TC6491K2

CK192837K

TC4205K2

TC7639K1

TC1750K2

TC4684K3

CK176583K1

TC4205K 1

CK176583K2

CK192299K3

TC5548K2

TC3519K2

- ТС8266K2

L TC12361K2

CK192837K2

TC6491K 4

TC5576K2

- TC4684K9

TC1750K7

TC727 1K3

- TC4684K5

- TC4684K4

TC1750K3

TC7271K2

- TC6492K3

- TC6491K

- TC5576K1

TC1750otK 1

TC4684K8

- TC4684K7

- TC7271K

- TC1750K5

TCA684KE

- TC4684K2

- TC1750K1

EU636772K1

TC185K5

CK192299K2

TC185K6

TC8213K2

TC8213K2 2

TC2049K2

TC13715K1

TC334K1

TC11158K 1

C5703K

TC8750K5

CV450668K3

TC13090K2

TC8508K2

TC13756K2

TC3973K3

TC1766K2

- TC4377K3

TC3823K2

TC10529K1

TC4377K2

TC3823K 1

TC185K2

CV450668K2

TC1766K1

TС 3973 K 1

TC6750K3

TC5750K1

TC3973K

TC6750K

TC6750K2

TC8508K

TC4377K1

TC5703K1

TC13090K1

TC3418K2

TC3419K1

TC8213K 1

TC2049K 1

- TC3418K

TCA754K

TC185K3

TC1212K-

TC5548K1

CV $450668 \mathrm{~K} 1$

TC6502K1

TC185K4

TC185K1

MONOGRINIAK

MONOGRIN1BK1

SAVIGNYGRNK

ORNIPARKGK1

ORNIPARK $10 K 1$
Clade I

Clade A

Clade II

Cade lic

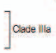

7

Clade lilib

Clade III

Cade lilc

Clade B

Clade IV

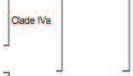

Clade V

Outgroup 


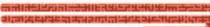

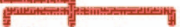

$$
100
$$

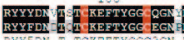

BYron

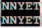

NNYDT
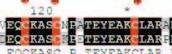

EOCRABC

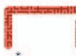

200

62

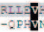

S.

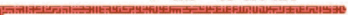
EI GE

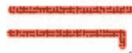

180

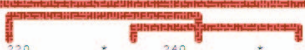

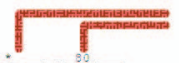

*
85

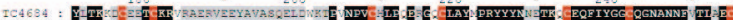
IC1750 : ${ }_{Y}^{4}$ TR

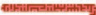
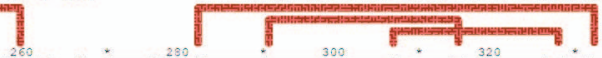

\section{0}

300

t.

340

ICACQ4: 2 RT

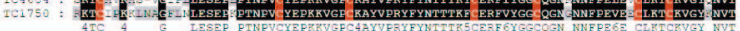

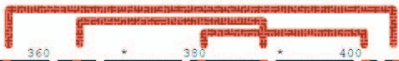

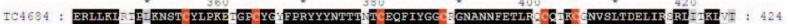

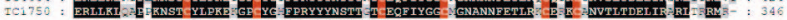

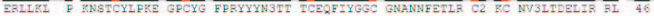

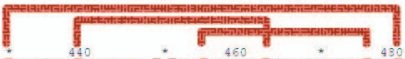

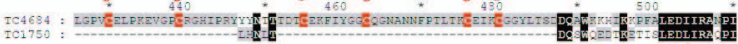

N I

DQ

IEL6IBA EI

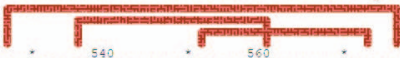

520

$T C 4684:$

TC1750:

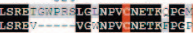

ISRE

\section{(6)}

GNGNNEYII

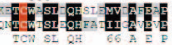

594 454

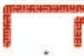

600

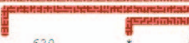

62.8

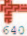

AYNEREFYNTLTKT EQFIYGE GGNANN

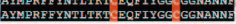

AYNERTFYNTLTKTCEQPIXGGCGGNANNF

GNGNIFVII

TC4684: FWF APPED TYGVDAGB FWI ADPEE TYSVDAGB FNP AEPETCRYSVAAGDS 
Figure 9

Ancestral Kunitz Domain

Domain
duplication

MRCA

TC4684

and

TC1750
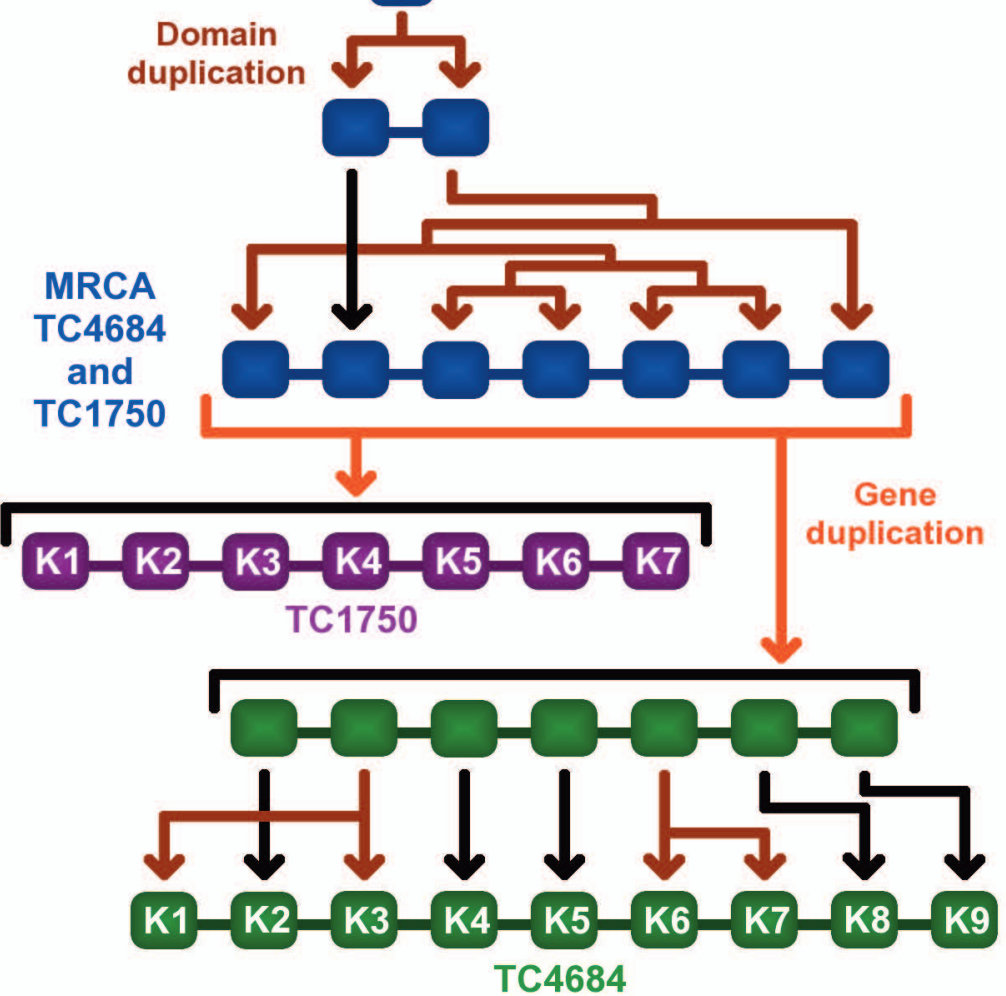
TC3519K1 TC8266K1 TC12361K1 TC6492K2 TC6492K4 TC6492K1 TC6491K2 CK192837K1 TC4205K2 TC7639K1 TC1750K TC4684K3 CK176583K1 TC4205K1 CK176583K2 CK192299K3 TC5 548K2 TC3519K2 TC $8266 \mathrm{~K} 2$ TC12361K2 CK192837K2 TC6491K4 TC5576K2 TC4684K9 TC1750K7 TC7271K3 TC4684K5 TC1750K4 TC4684K4 TC1750K3 TC7271K2 TC6492K3 TC6491K3 TC5576K1 TC1750K6 TC1750ptK1 TC4 684K8 TC4684K7 TC7271K1 TC1750K5 TC4684K6 TC4 684K2 TC1750K1 TC11274K1 EU636772K1 TC185K5 CK192299K2 TC185K 6 TC8213K2 CV4 42296K1 TC2049K2 TC13715K1 TC334K1

TC11158K1 TC5703K2 TC6750K5 CV450668K3 TC13090K2 TC8508K2 TC13756K2 TC3973K3 TC1766K2 TC4377K 3 TC3823K2 TC10529K1 TC4 377K2 TC3823K1 TC185K2 CV450668K2 TC1766K1 TC3973K1 TC6750K3 TC6750K1 TC3973K2 TC6750K 4 TC6750K2 TC8508K1 TC4 $377 \mathrm{~K} 1$ TC5703K1 TC13090K1 TC3418K2 TC3419K1 TC8213K1 TC2049K1 TC3418K1 TC4 754K1 TC185K3 TC1212K1 TC5548K1 CV450668K1 TC6502K1 TC185K4 TC185K1

MONOGRIN1AK MONOGRIN1BK SAVIGNYGRINK ORNIPARK 9K 1 ORNI PARK10K1

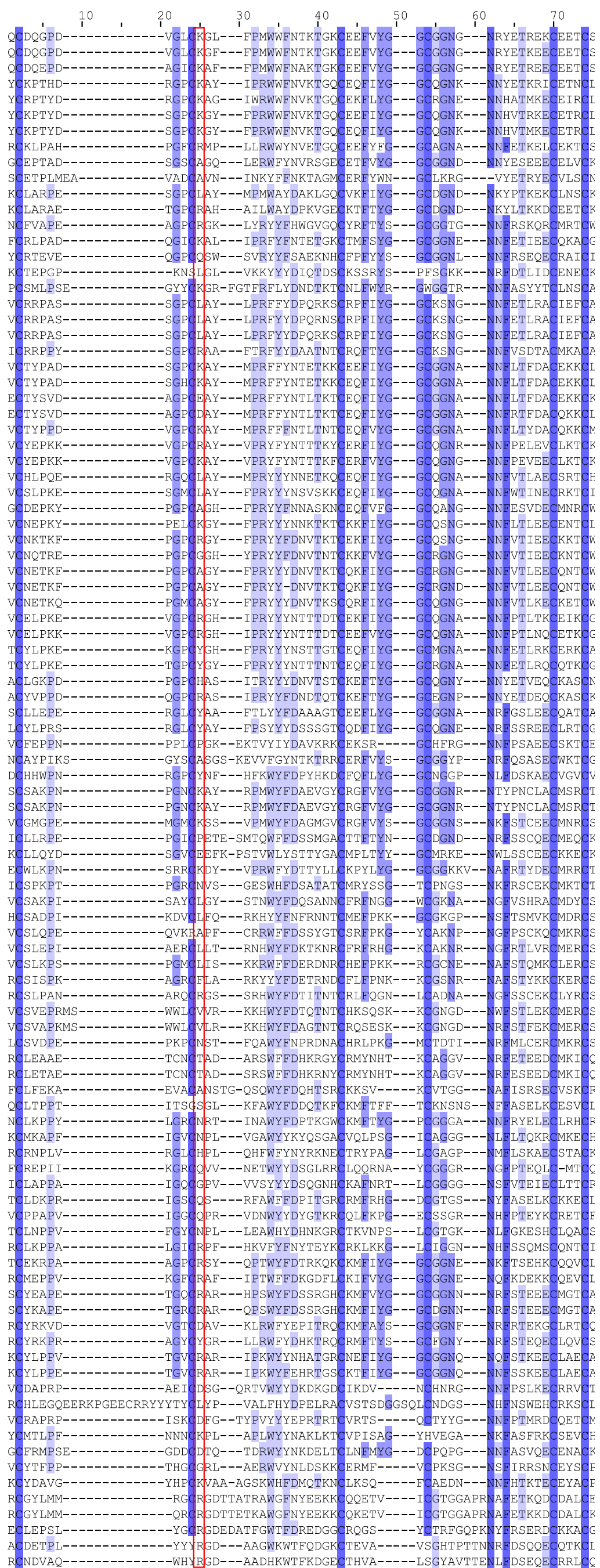



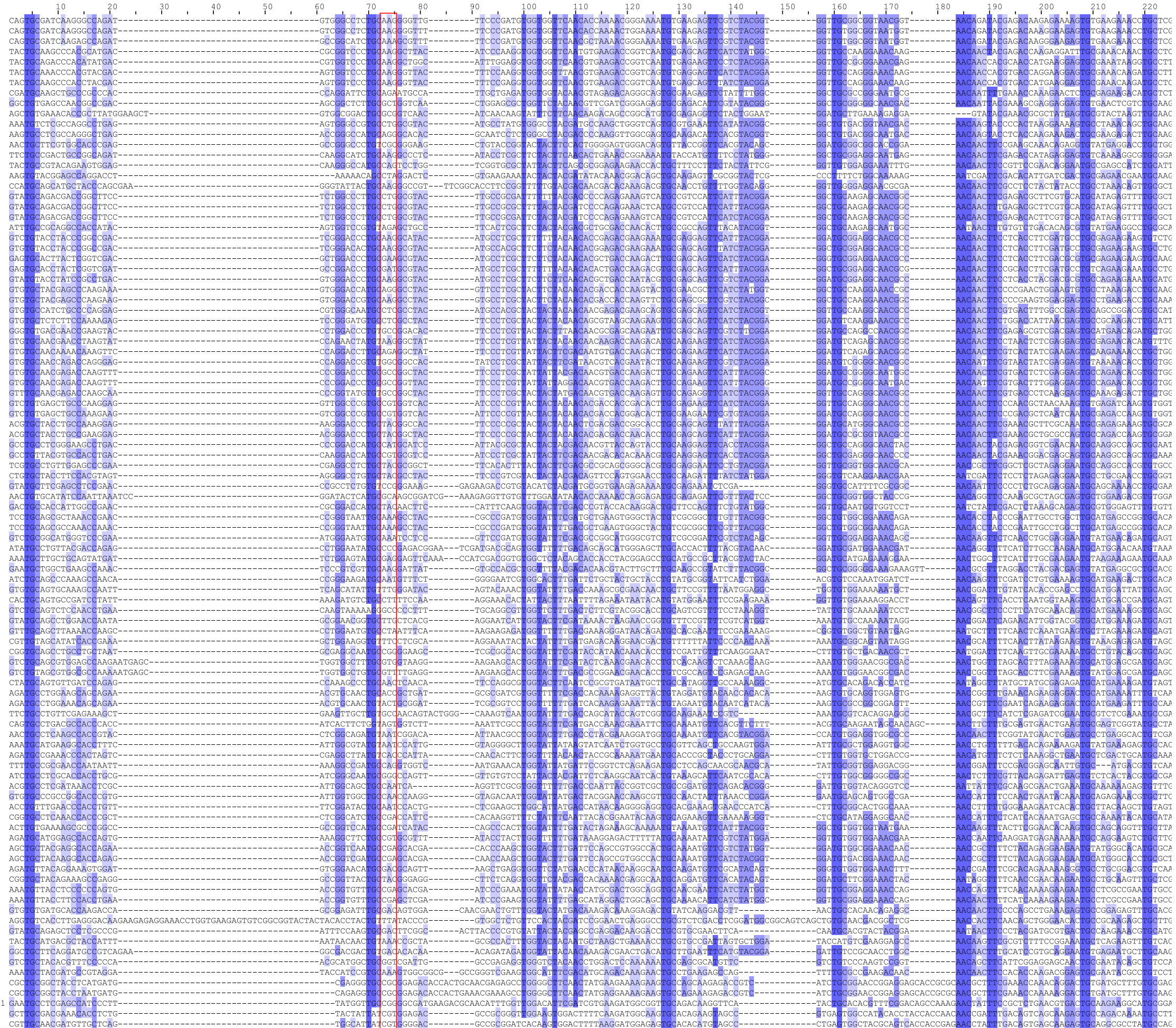
Table 1. Accession numbers of transcripts used in this study. The GenBank accession numbers of the amino acid sequences of transcripts used as queries (BPTI, TFPI, ixolaris and penthalaris) or as outgroups for phylogenetic studies (savignygrin, monogrin 1A and 1B, ORNIPARK9 and ORNIPARK 10) are shown.

\begin{tabular}{ll}
\hline Transcript Name & GenBank Accession \\
\hline Bovine pancreatic trypsin inhibitor (BPTI) & $\underline{\mathbf{1 1 5 1 1 4}}$ \\
Ixolaris & $\underline{\mathbf{1 5 0 7 7 0 0 2}}$ \\
Monogrin 1A & $\underline{\mathbf{1 1 4 1 5 2 9 6 6}}$ \\
Monogrin 1B & $\underline{\mathbf{1 1 4 1 5 2 9 6 8}}$ \\
ORNIPARK9 & $\underline{\mathbf{1 4 9 2 8 7 1 7 2}}$ \\
ORNIPARK10 & $\underline{\underline{\mathbf{1 4 9 2 8 6 8 9 8}}}$ \\
Penthalaris & $\underline{\underline{\mathbf{2 1 6 4 2 6 6}}}$ \\
Savignygrin & $\underline{\mathbf{2 1 4 3 5 9 8 1}}$ \\
Tissue factor pathway inhibitor (TFPI) & $\underline{\mathbf{1 2 5 9 3 2}}$ \\
\hline
\end{tabular}


Table 2. Taxon set calibration points used and recovered. Three taxon sets (Ixodida, Argasidae and Metastriate) were defined with divergence times as calculated by Jeyaprakash and Hoy (2009). The mean divergence times that were recovered for these taxa during phylogenetic analysis are shown.

\begin{tabular}{ccc}
\hline Taxon Sets & Calibration Points & Recovered Divergence Times \\
& MYA \pm SD (95\% CI) & MYA \pm SD (95\% HPD) \\
\hline Ixodida & $300 \pm 27(245,351)$ & $255.3 \pm 0.9(204.9,310.2)$ \\
Argasidae & $214 \pm 28(160,269)$ & $213.9 \pm 1.5(154.5,272.7)$ \\
Metastriate & $134 \pm 22(95,181)$ & $164.9 \pm 0.4(127.4,204.2)$ \\
\hline
\end{tabular}

MYA, million years ago; CI, confidence interval; HPD, highest posterior density interval. 
Table 3. Recent Kunitz domain duplications. Of the nine sub-clades that were studied to find domain duplications (Fig. 6), seven sub-clades had sequences that were recently duplicated, indicated by an amino acid identity of more than $85 \%$ and a nucleotide identity of at least $90 \%$. In this manner, eight groups of sequences that showed recent duplication were identified and the divergence times, amino acid identity and similarity as well as the nucleotide identity within these groups are shown.

\begin{tabular}{|c|c|c|c|c|c|}
\hline Sub-Clade & $\begin{array}{c}\text { Kunitz } \\
\text { Domain }\end{array}$ & $\begin{array}{c}\text { Divergence } \\
\text { time } \\
\text { (MYA) }\end{array}$ & $\begin{array}{c}\text { Amino acid } \\
\text { identity }\end{array}$ & $\begin{array}{c}\text { Amino acid } \\
\text { similarity }\end{array}$ & $\begin{array}{c}\text { Nucleotide } \\
\text { identity }\end{array}$ \\
\hline \multirow{3}{*}{ Clade Ia } & TC3519K1 & \multirow{3}{*}{9.86} & \multirow{3}{*}{$86 \%-94 \%$} & \multirow{3}{*}{$92 \%-100 \%$} & \multirow{3}{*}{$91 \%-94 \%$} \\
\hline & TC8266K1 & & & & \\
\hline & TC12361K1 & & & & \\
\hline Clade Ib & $\begin{array}{l}\text { TC6491K2 } \\
\text { TC6492K1 }\end{array}$ & 4.73 & $96 \%$ & $98 \%$ & $94 \%$ \\
\hline \multirow{3}{*}{ Clade IIa } & TC3519K2 & \multirow{3}{*}{3.56} & \multirow{3}{*}{$96 \%-98 \%$} & \multirow{3}{*}{$98 \%-100 \%$} & \multirow{3}{*}{$96 \%$} \\
\hline & TC8266K2 & & & & \\
\hline & TC12361K2 & & & & \\
\hline Clade IIb & $\begin{array}{l}\text { TC5576K2 } \\
\text { TC6491K4 }\end{array}$ & 8.62 & $96 \%$ & $98 \%$ & $92 \%$ \\
\hline \multirow{2}{*}{ Clade IIc } & $\begin{array}{l}\text { TC1750K4 } \\
\text { TC4684K5 }\end{array}$ & 9.82 & $88 \%$ & $96 \%$ & $90 \%$ \\
\hline & $\begin{array}{c}\text { TC1750K6 } \\
\text { TC1750ptK1 }\end{array}$ & 2.27 & $96 \%$ & $96 \%$ & $94 \%$ \\
\hline Clade IIIa & $\begin{array}{c}\text { CV442296K1 } \\
\text { TC8213K2 }\end{array}$ & 1.05 & $100 \%$ & $100 \%$ & $96 \%$ \\
\hline Clade IVa & $\begin{array}{l}\text { TC3418K2 } \\
\text { TC3419K1 }\end{array}$ & 11.38 & $90 \%$ & $98 \%$ & $93 \%$ \\
\hline
\end{tabular}

MYA, million years ago. 
Graphical Abstract

\section{Rhipicephalus}

\section{microplus}

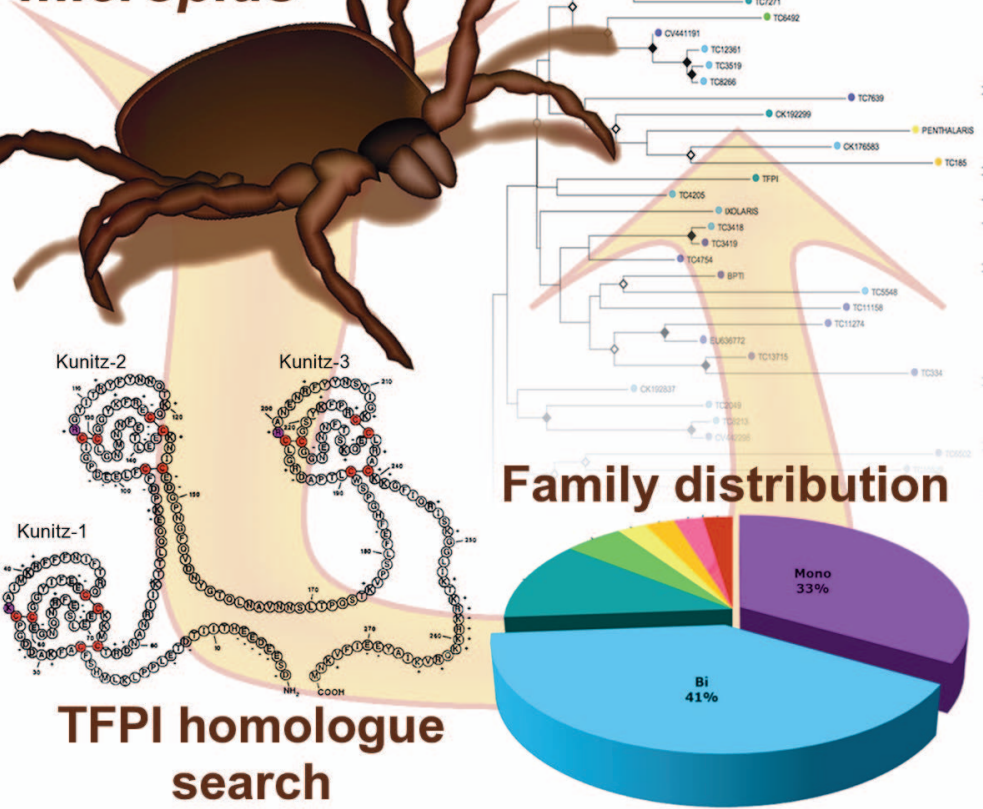

